\title{
Acidentes causados por aranhas e escorpiões no Estado do Ceará, Nordeste do Brasil: casos subnotificados e superestimados baseados na distribuição geográfica das espécies
}

\author{
Raul Azevedo ${ }^{1}$, Francisco Roberto de Azevedo ${ }^{1}$, Relrison Dias Ramalho ${ }^{2}$, Paulo \\ André Margonari Goldoni ${ }^{3} \&$ Antonio Domingos Brescovit ${ }^{3}$
}

(1) Universidade Federal do Cariri - Campus Crato, Centro de Ciências Agrárias e da Biodiversidade, Laboratório de Entomologia, Rua Ícaro de Sousa Moreira, Barro Branco, Crato 63130-025, Ceará, Brasil. E-mail: raulbiologo@gmail.com, roberto.azevedo@ufca.edu.br

(2) Secretaria da Saúde do Estado do Ceará (SESA), Núcleo de Controle de Vetores, Av. Almirante Barroso, 600, Praia de Iracema, Fortaleza 60060-440, Ceará, Brasil. E-mail: relrisondias@gmail.com

(3) Fundação Butantan, Instituto Butantan, Seção de Artrópodes Peçonhentos, Laboratório de Artrópodes, Av. Vital Brasil 1500, Butantã 05503-900, São Paulo, Brasil. E-mail: paulo.goldoni@butantan.gov.br, antonio.brescovit@butantan.gov.br

Azevedo R., Azevedo F.R., Ramalho R.D., Goldoni P.A.M. \& Brescovit A.D. (2017) Acidentes causados por aranhas e escorpiões no Estado do Ceará, Nordeste do Brasil: casos subnotificados e superestimados baseados na distribuição geográfica das espécies. Pesquisa e Ensino em Ciências Exatas e da Natureza, 1(2): 144-158.

Resumo: A frequência dos acidentes causados por aracnídeos tem crescido ao longo dos anos e a maioria dos acidentes reportados não apresenta identificação das espécies causadoras, contribuindo assim para a subnotificação dos dados. No Estado do Ceará, informações específicas sobre acidentes com notificações completas ou descrição dos acidentes são escassos. No presente trabalho, os dados sobre acidentes com aranhas e escorpiões no Estado do Ceará do período de 2010 a 2015 foram confrontados com a distribuição geográfica baseada em dados existentes nas principais coleções científicas brasileiras de aracnídeos. Foi observado um aumento do número de acidentes causados por aranhas e escorpiões, principalmente em 2013 para escorpiões e um constante número de municípios reportaram acidentes escorpiônicos para o período. De acordo com o Sistema de Informação de Agravos de Notificação - SINAN, no Estado do Ceará, muitos casos foram atribuídos a Phoneutria sp., Loxosceles sp. e Latrodectus sp., contudo essa ocorrência não está correlacionada com sua distribuição geográfica existente nas coleções de aracnídeos. Desse modo, o conhecimento da distribuição geográfica das espécies de interesse em saúde combinados com registros devidamente notificados podem contribuir para redução dos números de acidentes e para o desenvolvimento de políticas voltadas para saúde pública.

Palavras chave: Araneismo, Escorpionismo, Casos Notificados, Nordeste Brasileiro.

\section{Accidents caused by spiders and scorpions in the state of Ceará, northeastern Brazil: Under-notified and overestimated cases based on the geographic distribution of the species}

Abstract: The accidents caused by arachnids have increased its frequency along the years and the majority of accidents reports do not contain species identification, contributing to underreporting of data. In Ceará state, specific information about accidents with full notifications or accident description are scarce. In this present study, data about accidents involving spiders and scorpions in Ceará state from 2010 to 2015 period were correlated with geographic distribution based on data from main Brazilian scientific collections of arachnids. An increased number of accidents caused by spiders and scorpions was observed, mainly in 2013 for scorpions, and a constant number of municipalities reported scorpionic accidents to this period. According to Sistema de Informação de Agravos de Notificação - SINAN, in Ceará state, many cases were credited to Phoneutria sp., Loxosceles sp., and Latrodectus sp., whoever this occurrence is not correlated 
with their geographic distribution supported on arachnid collections data. Therefore, knowledge on species geographic distribution with public health significance combined with properly notified records can contribute to reduction of accidents numbers and development of politics aimed to public health.

Key words: Araneism, Scorpionism, Underreporting cases, Northeast Brazil.

\section{Introdução}

Atualmente existem 46.912 espécies de aranhas (World Spider Catalog 2017) e 2.239 espécies de escorpiões descritas (Rein 2012). Desse total, no território brasileiro ocorrem três gêneros de aranhas e um de escorpião responsáveis por causar acidentes em humanos (BRASIL 2001), respectivamente: Latrodectus Walckenaer, 1805; Loxosceles Heineken \& Lowe, 1832; Phoneutria Perty, 1833; e Tityus Koch, 1836. Contudo, para os escorpiões, referências mais atuais citam ainda outros gêneros de escorpiões que causam acidentes, porém de menor relevância para a saúde pública, tais como: Ananteris Thorell, 1891; Rhopalurus Thorell, 1876; Bothriurus Peters, 1861; Thestylus Simon, 1880 e Brotheas C.L. Kock, 1837 (BRASIL 2009; Brazil \& Porto 2010).

O Estado do Ceará (034'02" S, 38²32'35" O) está inserido no Bioma Caatinga e muitas de suas áreas apresentam elevada temperatura anual (Reis 1976) e baixa ou pluviosidade inexistente (Nimer 1972). O Estado do Ceará é composto por 184 municípios e $75 \%$ de sua população vive em áreas urbanas (IBGE 2010).

Os casos de acidentes causados por aracnídeos têm crescido ao longo dos últimos anos assim como o número do agente causador não identificado (Camplesi et al. 2014), contribuindo para os casos subnotificados. O número de acidentes tem crescido constantemente, de modo que o Brasil apresenta um dos maiores números de acidentes no mundo (Lourenço \& Eickstead 2003), e hoje, a situação epidemiológica dos acidentes com aranhas e escorpiões contabilizam mais de 359.000 acidentes causados para aranhas e mais 800.000 acidentes causados por escorpiões do período de 2000 a 2016 (Acidentes por animais peçonhentos 2017).

Ao analisar os dados do Sistema de Informação de Agravos de Notificação (SINAN) para o Estado do Ceará, percebe-se o aumento constante do número de acidentes e de municípios que reportam acidentes causados por aranhas e escorpiões (SINAN, 2017), bem como observa-se a ocorrência de três gêneros de aranhas de interesse em saúde e somente a menção a acidentes causados por escorpiões, sem a informação da espécie causadora. Especificamente, o número de acidentes causados por aranhas e escorpiões tem crescido ao longo dos anos, principalmente em suas áreas mais populosas (Furtado et al. 2016; SINAN 2017). Apesar dos dados do SINAN não fazerem menção à espécies de escorpiões para o Estado, a literatura reporta que Tityus stigmurus (Thorell, 1876) e T. serrulatus Lutz \& Mello, 1922 são os principais causadores de acidentes na cidade Fortaleza (capital do Estado) durante o período de 2003-2004 (Alves et al. 2007).

Portanto, o objetivo do presente trabalho é correlacionar a evolução dos acidentes causados por aranhas e escorpiões no Estado do Ceará por meio dos dados oriundos do SINAN com a distribuição geográfica baseada nos dados existentes nas coleções científicas de aracnídeos no Brasil, analisando potenciais dados superestimados.

\section{Material e Métodos}

\section{Acidentes causados por aracnídeos no Estado do Ceará}

Os números de acidentes causados no Estado do Ceará foram obtidos por meio do banco de dados do SINAN (2017) e na literatura. Eles foram sintetizados em Macrorregiões de Saúde (Figura 1) delimitadas de acordo com a Secretaria de Saúde do Estado do Ceará (Ceará 2010). 


\section{MAPA DO CEARÁ (MACRORREGIÕES)}

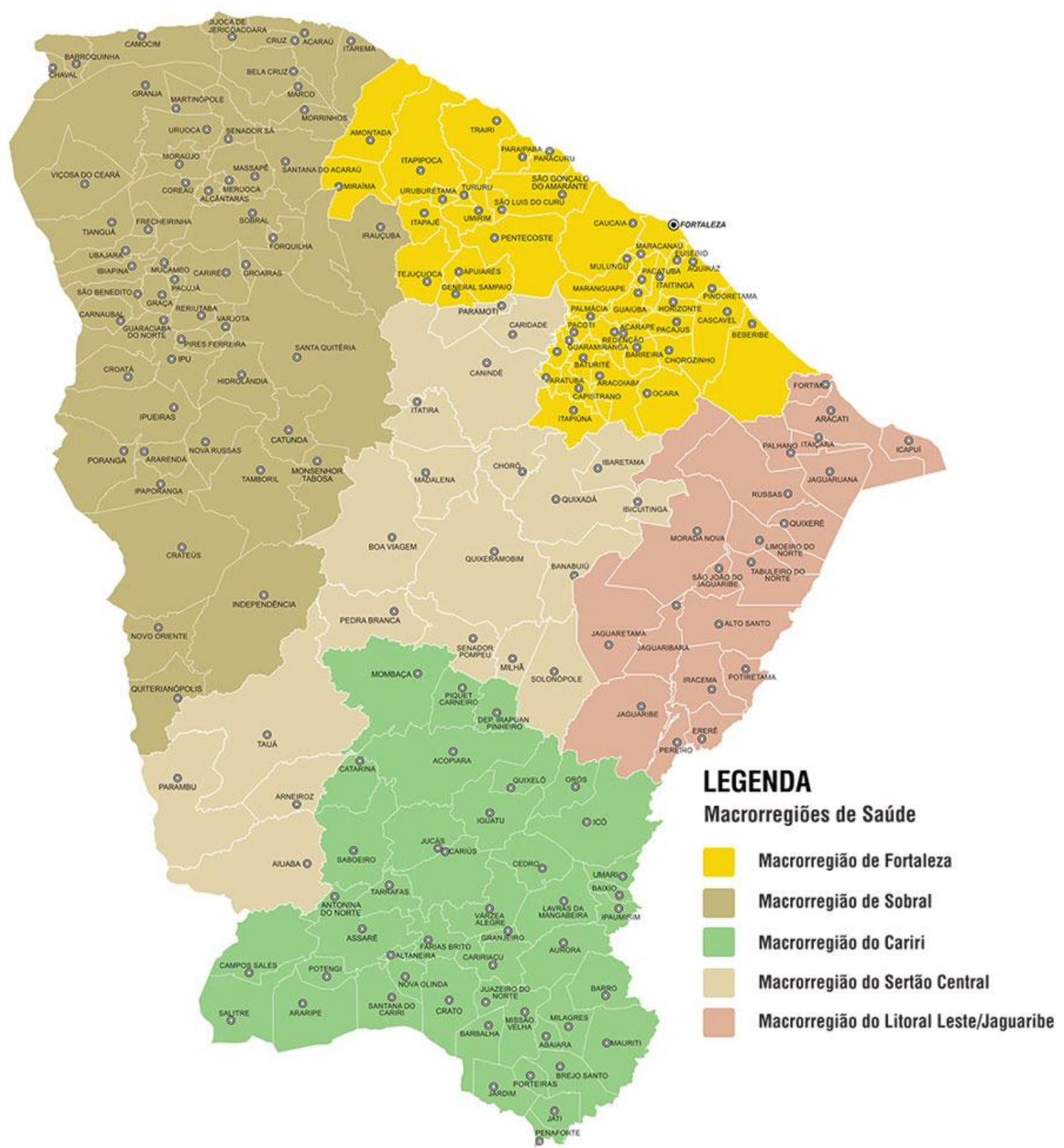

Figura 1. Mapa das macrorregiões de saúde do Estado do Ceará baseado na delimitação da Secretaria de Saúde do Estado do Ceará (Ceará 2010).

A tabulação das espécies de aranhas de interesse em saúde seguiu a classificação do SINAN (Phoneutria, Loxosceles e Latrodectus). Os dados relativos a escorpiões foram considerados como atribuídos a Tityus, apesar do SINAN não fazer nenhum tipo de classificação.

As informações dos prontuários dos pacientes que sofreram acidentes com animais peçonhentos são transferidas para as fichas de investigação para a notificação de acidentes que é encaminhada para as Secretarias de Saúde. Por sua vez, esses dados são transmitidos para o Ministério da Saúde, então, disponibilizados para o SINAN.

Os municípios que compõem cada macrorregião e seus números de habitantes são expressos na Tabela 1. Os dados são delimitados de acordo com a Secretaria de Saúde do Estado do Ceará (Ceará 2010). 
Tabela 1. Macrorregiões, respectivos municípios e número de habitantes (expressos entre parênteses) baseado na delimitação da Secretaria de Saúde do Estado Ceará (Ceará 2010).

\begin{tabular}{|c|c|}
\hline Macrorregião & Municípios \\
\hline Fortaleza & $\begin{array}{l}\text { Fortaleza (2.551.805), Acarape (16.011), Amontada (41.227), Apuiarés } \\
\text { (14.397), Aquiraz (76.186), Aracoiaba (25.988), Aratuba (11.482), Barreira } \\
\text { (20.371), Baturité (34.512), Capistrano (17.470), Caucaia (34.493), Euzébio } \\
\text { (49.455), General Sampaio (6.591), Guaiuba (25.310), Guaramiranga (3.909), } \\
\text { Itaitinga (37.705), Itapagé (50.211), Itapipoca (122.220), Itapiuna (19.409), } \\
\text { Jaguaretama (18.040), Jaguaruana (33.174), Maracanaú (217.922), } \\
\text { Maranguape (120.405), Miraíma (13.259), Morada Nova (62.287), Mulungu } \\
\text { (12.196), Pacatuba (77.723), Pacoti (11.857), Palhano (9.126), Palmácia } \\
\text { (12.624), Paracuru (32.919), Paraipaba (31.413), Pentecoste (36.442), Redenção } \\
\text { (27.088), Russas (73.436), São Gonçalo do Amarante (46.247), São Luiz do } \\
\text { Curu (12.663), Tejussuoca (18.083), Trairí (53.561), Tururu (15.224), Umirim } \\
\text { (19.349) e Uruburetama (20.768). Total de municípios: 42. }\end{array}$ \\
\hline Sertão Central & $\begin{array}{l}\text { Aiuaba (16.784), Arneiroz (7.766), Banabuiu (17.775), Boa Viagem (53.608), } \\
\text { Canindé (76.439), Caridade (21.236), Choró (13.195), Ibaretama (13.155), } \\
\text { Ibicuitinga (11.890), Itatira (19.861), Madalena (19.017), Milhã (13.207), } \\
\text { Parambu (31.462), Paramoti (11.517), Pedra Branca (42.643), Quixadá } \\
\text { (83.990), Quixeramobim (75.565), Senador Pompeu (26.656), Solonópole } \\
\text { (18.025) e Tauá (57.246). Total de municípios: } 20 \text {. }\end{array}$ \\
\hline Sobral & $\begin{array}{l}\text { Ararenda (10.723), Alcântaras (11.171), Acaraú (60.137), Cariré (18.629), } \\
\text { Barroquinha (14.771), Bela Cruz (31.804), Camocim (61.918), Carnaubal } \\
\text { (17.282), Catunda (10.218), Chaval (12.865), Coreaú (22.653), Crateús (74.103), } \\
\text { Croatá (17.569), Cruz (23.344), Forquilha (22.998), Frecheirinha (13.402), } \\
\text { Graça (15.281), Granja (53.435), Groairas (10.668), Guaraciaba do Norte } \\
\text { (38.832), Hidrolândia (19.882), Ibiapina (24.458), Independência (25.946), } \\
\text { Ipaporanga (11.500), Ipú (41.190), Ipueiras (38.159), Irauçuba (23.202), } \\
\text { Itarema (39.494), Jijoca de Jericoacoara (18.292), Marco (25.944), Martinópole } \\
\text { (10.693), Massapê (36.854), Meruoca (14.377), Monsenhor Tabosa (16.984), } \\
\text { Moraújo (8.393), Morrinhos (21.561), Mucambo (14.335), Nova Russas } \\
\text { (31.692), Novo Oriente (28.075), Pacujá (6.131), Pires Ferreira (10.556), } \\
\text { Poranga (12.203), Quiterianópoles (20.505), Reriutaba (19.281), Santa Quitéria } \\
\text { (43.358), Santana do Acaraú (31.133), São Benedito (45.653), Senador Sá } \\
\text { (7.210), Sobral (197.663), Tamboril (25.675), Tianguá (72.110), Ubajara } \\
\text { (33.205), Uruoca (13.348), Varjota (18.024) e Viçosa do Ceará (57.719). Total } \\
\text { de municípios: } 55 \text {. }\end{array}$ \\
\hline Litoral Leste / Jaguaribe & $\begin{array}{l}\text { Alto Santo (16.767), Aracati (71.749), Beberibe (51.442), Cascavel (68.926), } \\
\text { Chorozinho (19.187), Ererê (7.041), Fortim (15.603), Horizonte (60.584), Icapuí } \\
\text { (19.129), Iracema (14.011), Itaiçaba (7.567), Jaguaribara (10.892), Jaguaribe } \\
\text { (34.683), Limoeiro do Norte (57.372), Ocara (24.829), Pacajús (66.510), Pereiro } \\
\text { (16.063), Pindoretama (19.733), Potiretama (6.278), Quixeré (19.772), São João } \\
\text { do Jaguaribe (7.829) e Tabuleiro do Norte (30.018). Total de municípios: } 22 \text {. }\end{array}$ \\
\hline Cariri & $\begin{array}{l}\text { Abaiara (11.089), Acopiara (52.661), Altaneira (7.196), Antonina do Norte } \\
\text { (7.172), Araripe (21.170), Assaré (22.988), Aurora (24.716), Baixio (6.165), } \\
\text { Barbalha (57.818), Barro (22.104), Brejo Santo (47.218), Campos Sales } \\
\text { (27.030), Caririaçu (26.821), Cariús (18.815), Catarina (19.676), Cedro (24.958), } \\
\text { Crato (126.591), Deputado Irapuan Pinheiro (9.360), Farias Brito (19.015), } \\
\text { Granjeiro (4.565), Icó (66.885), Iguatu (100.053), Ipaumirim (12.256), Jardim } \\
\text { (27.067), Jati (7.764), Juazeiro do Norte (261.289), Jucás (24.351), Lavras da } \\
\text { Mangabeira (31.435), Mauriti (45.640), Milagres (28.487), Missão Velha } \\
\text { (35.056), Mombaça (43.493), Nova Olinda (14.908), Orós (21.503), Penaforte } \\
\text { (8.666), Piquet Carneiro (16.169), Porteiras (15.108), Potengi (10.651), Quixelô } \\
\text { (15.046), Saboeiro (15.835), Salitre (15.976), Santana do Cariri (17.445), } \\
\text { Tarrafas (8.949), Umari (7.660) e Várzea Alegre (39.651). Total de municípios: } \\
\text { 45. }\end{array}$ \\
\hline
\end{tabular}




\section{Registro das aranhas e escorpiões de interesse em saúde pública para o Estado do Ceará}

As informações sobre as espécies citadas e respectiva distribuição geográfica foram obtidas a partir do material depositado nas seguintes coleções de aracnídeos: Instituto Butantan São Paulo (IBSP), curador - Dr. Antônio Domingos Brescovit; Universidade Federal de Minas Gerais (UFMG), curador - Dr. Adalberto José dos Santos e Museu Nacional do Rio de Janeiro (MNRJ), curador - Dr. Adriano Brilhante Kury.

As espécies citadas foram identificadas por comparação do material depositado na coleção do IBSP e MNRJ, e também por meio das chaves de classificação para gêneros: Phoneutria (Martins \& Bertani 2007); Loxosceles (Gertsch 1967); Latrodectus (Levi 1959) e Tityus (De Souza et al. 2009).

\section{Resultados}

\section{Evolução dos Acidentes de 2010 a 2015}

Foi observado um aumento do número de acidentes causados por aranhas e escorpiões em cada macrorregião do Estado do Ceará de 2010 a 2015 (Figura 2A-B). A macrorregião Fortaleza apresentou os maiores números de acidentes de escorpiões no ano de 2013 (Figura 2A).

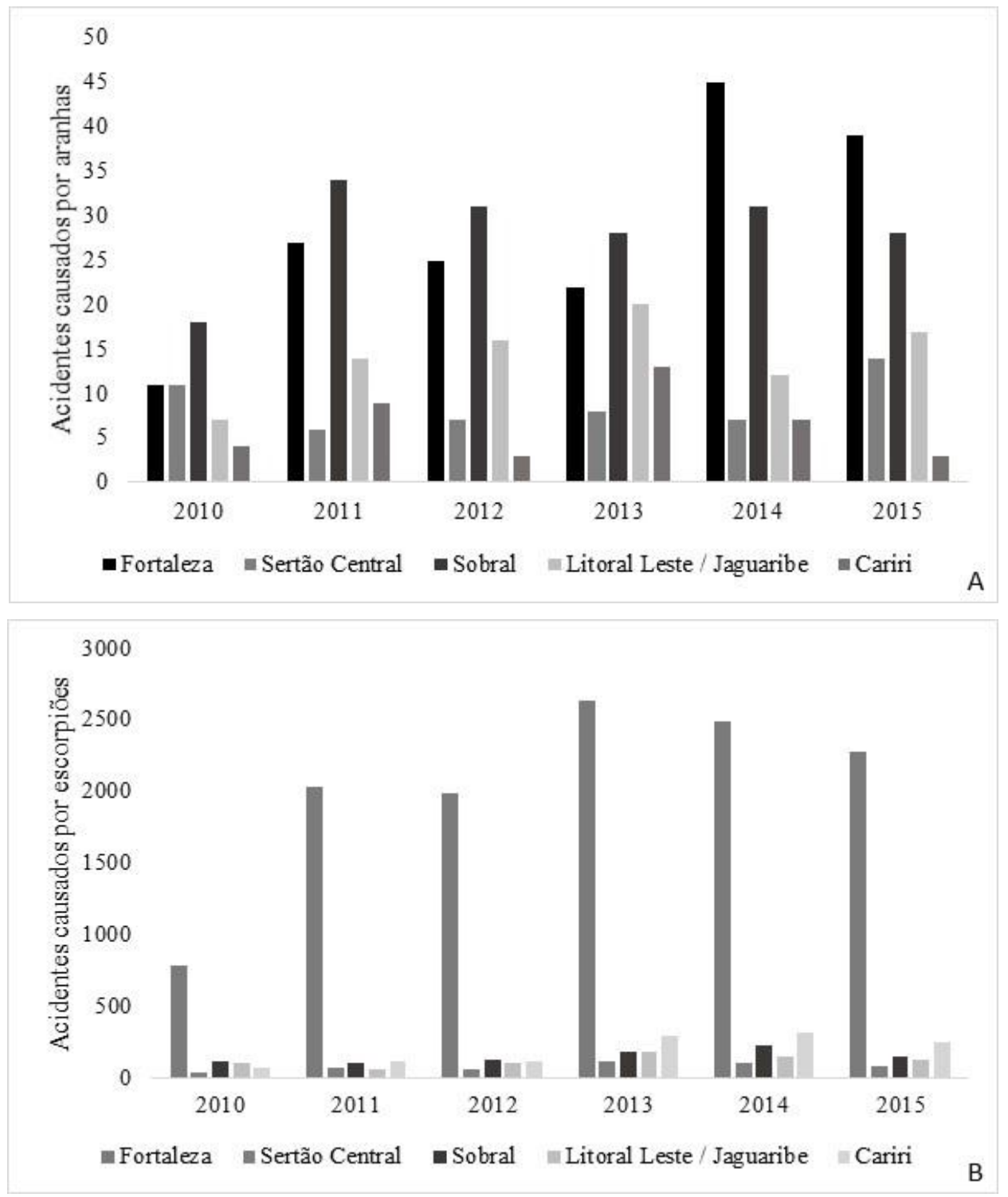

Figura 2. Número de Acidentes causados por aranhas (A) e escorpiões (B) por macrorregião do Estado do Ceará para o período de 2010-2015. 
Não foi observado aumento no número de municípios que reportaram acidentes causados por aranhas (Figura 3A), contudo tendência oposta foi observada para escorpiões, principalmente em 2015 (Figura 3B).
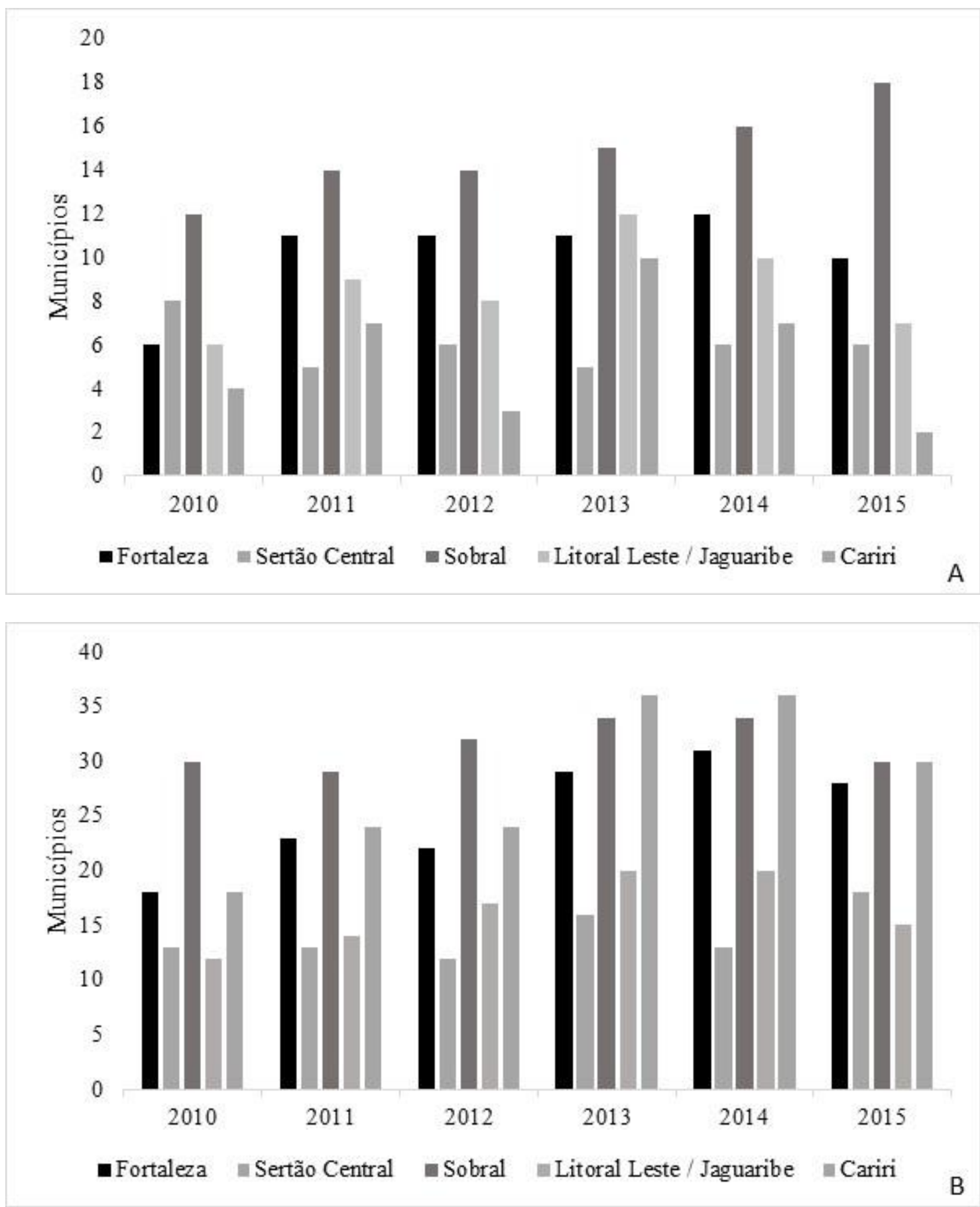

Figura 3. Número de Municípios que reportaram acidentes causados por aranhas (A) e escorpiões (B) por macrorregiões para o período de 2010-2015.

Os dados do SINAM citam espécies causadoras de acidentes identificadas somente em categoria taxonômica de gênero (Tabela 2). De acordo com as informações do SINAM, todos os gêneros de interesse médico ocorrem no Estado do Ceará. Loxosceles foi o gênero mais reportado dentre os municípios, seguido por Phoneutria e por último Latrodectus (Tabela 2). 
Tabela 2. Acidentes atribuídos à Phoneutria sp., Loxosceles sp., Latrodectus sp. e Tityus sp. para os municípios do Estado do Ceará durante o período de 2010 a 2015 baseado em dados obtidos do SINAN. Números de acidentes expressos entre parêntesis.

\begin{tabular}{|c|c|}
\hline Espécies & Anos / Municípios \\
\hline Phoneutria sp. & $\begin{array}{l}2010 \text { - Aratuba (1); Tianguá (1); } 2011 \text { - Baturité (1); Boa Viagem (1); Jaguaruana (1); } \\
\text { Marco (1); } 2012 \text { - Banabuiú (1); Fortaleza (1); Icapuí (1); Ipueiras (1); Martinópole (1); } \\
\text { Reriutaba (1); } 2013 \text { - Bela Cruz (1); Cascavel (1); Fortaleza (1); Ipueiras (1); Maracanaú } \\
\text { (1); Pereiro (1); } 2014 \text { - Croatá (1); Fortaleza (1); Russas (1); } 2015 \text { - Independência (1); } \\
\text { Orós (1). }\end{array}$ \\
\hline Loxosceles sp. & $\begin{array}{l}2010 \text { - Aracati (1); Aracoiaba (1); Beberibe (1); Fortaleza (1); Guaraciaba do Norte (1); } \\
\text { Irauçuba (2); Maracanaú (1); Quixeramobim (1); Russas (2); Sobral (1); } 2011 \text { - } \\
\text { Aracoiaba (1); Beberibe (1); Crateús (1); Fortaleza (5); Guaraciaba do Norte (1); } \\
\text { Ipueiras (2); Itaiçaba (1); Itarema (1); Milagres (1); Morada Nova (1); Russas (2); Sobral } \\
\text { (1); } 2012 \text { - Bela Cruz (1); Carnaubal (1); Coreaú (1); Fortaleza (3); Guaraciaba do Norte } \\
\text { (1); Ipu (2); Ipueiras (4); Jaguaruana (1); Reriutaba (1); Sobral (2); Tabuleiro do Norte } \\
\text { (1); Tauá (1); } 2013 \text { - Aracati (1); Aracoiaba (1); Crato (1); Croatá (2); Fortaleza (1); } \\
\text { Fortim (1); Horizonte (1); Ibiapina (1); Icapuí (1); Irauçuba (1); Jaguaribe (1); } \\
\text { Jaguaruana (1); Juazeiro do Norte (1); Pacajús (1); Quixelô (1); Trairi (1); } 2014 \text { - Bela } \\
\text { Cruz (1); Croatá (1); Fortaleza (9); Fortim (1); Ibaretama (1); Ibiapina (1); Ipueiras (1); } \\
\text { Jaguaruana (1); São Benedito (1); Uruburetama (1); 2015 - Aracati (1); Barroquinha } \\
\text { (1); Fortaleza (4); Itapipoca (1); Quiterianópolis (1). }\end{array}$ \\
\hline Latrodectus sp. & $\begin{array}{l}2011 \text { - Aracati (1); Santana do Cariri (1); } 2012 \text { - Aiuaba (1); Fortaleza (1); } 2013 \text { - } \\
\text { Milagres (1); } 2014 \text { - Boa Viagem (1); Fortaleza (2); } 2015 \text { - Fortaleza (4). }\end{array}$ \\
\hline Tityus sp. & 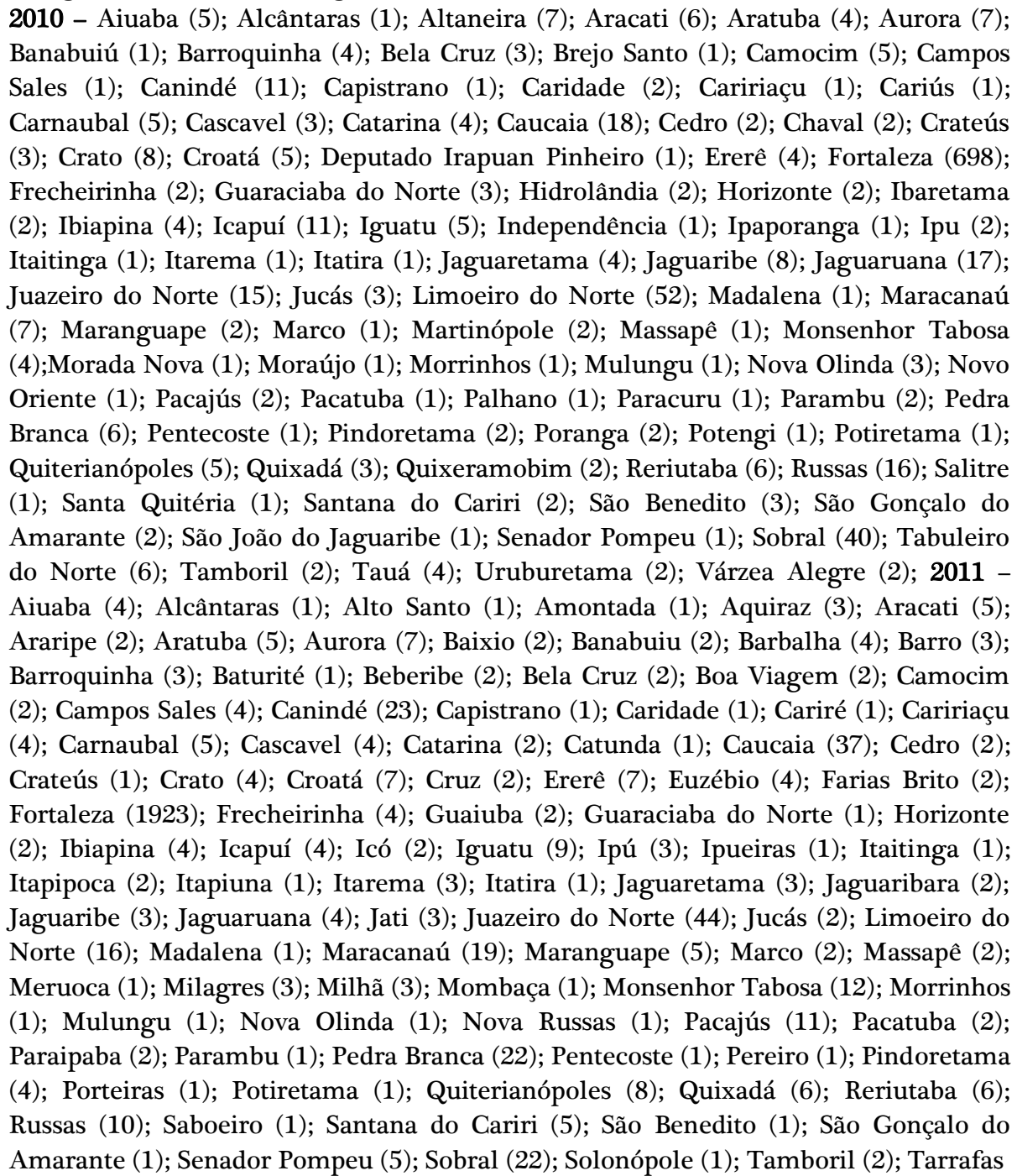 \\
\hline
\end{tabular}


Tabela 2. Acidentes atribuídos à Phoneutria sp., Loxosceles sp., Latrodectus sp. e Tityus sp. para os municípios do Estado do Ceará durante o período de 2010 a 2015 baseado em dados obtidos do SINAN. Números de acidentes expressos entre parêntesis (continuação).

\begin{tabular}{|c|c|}
\hline Espécies & Anos / Municípios \\
\hline Tityus sp. & 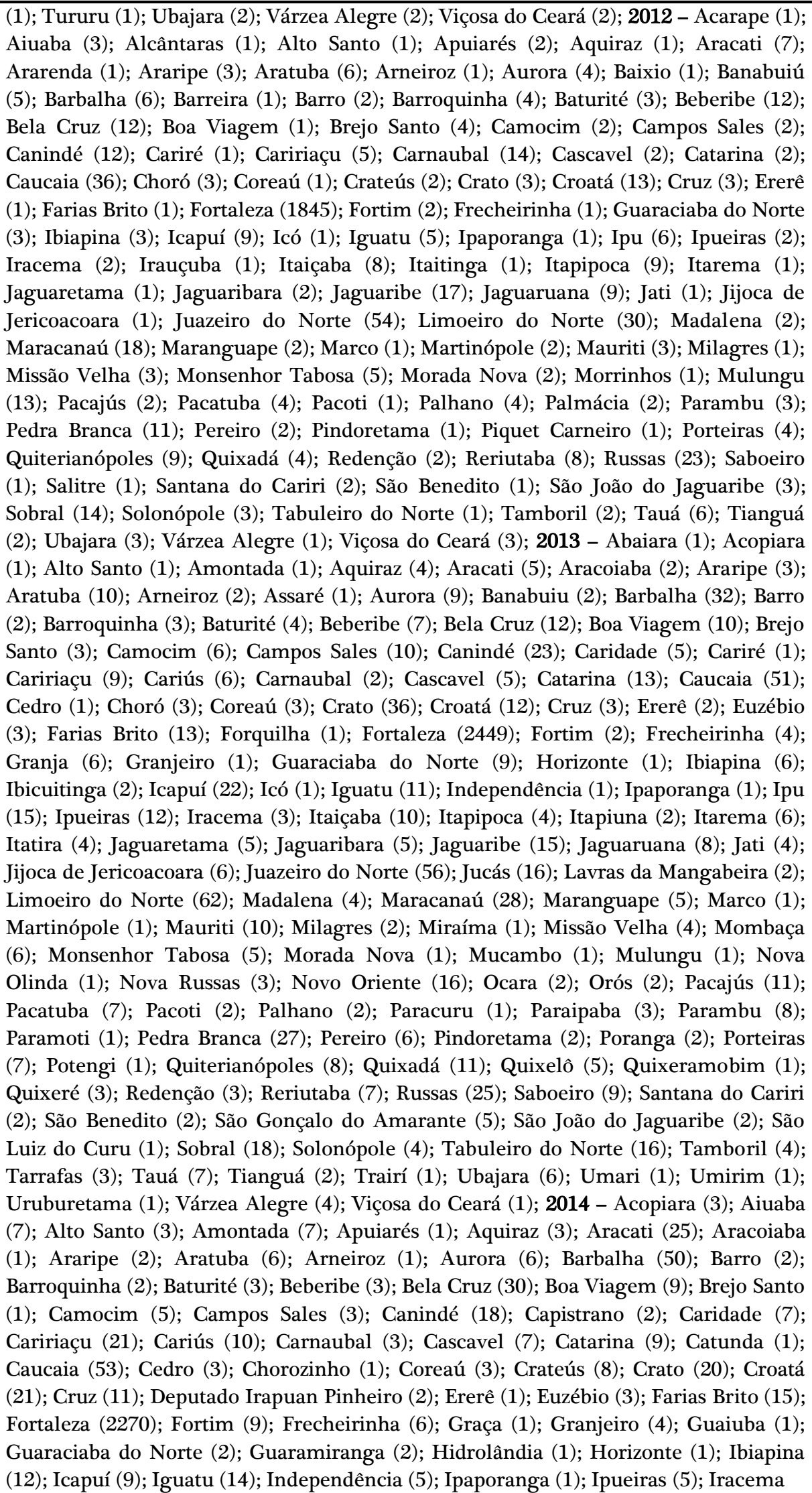 \\
\hline
\end{tabular}


Tabela 2. Acidentes atribuídos à Phoneutria sp., Loxosceles sp., Latrodectus sp. e Tityus sp. para os municípios do Estado do Ceará durante o período de 2010 a 2015 baseado em dados obtidos do SINAN. Números de acidentes expressos entre parêntesis (continuação).

\begin{tabular}{|c|c|}
\hline Espécies & Anos / Municípios \\
\hline Tityus sp. & 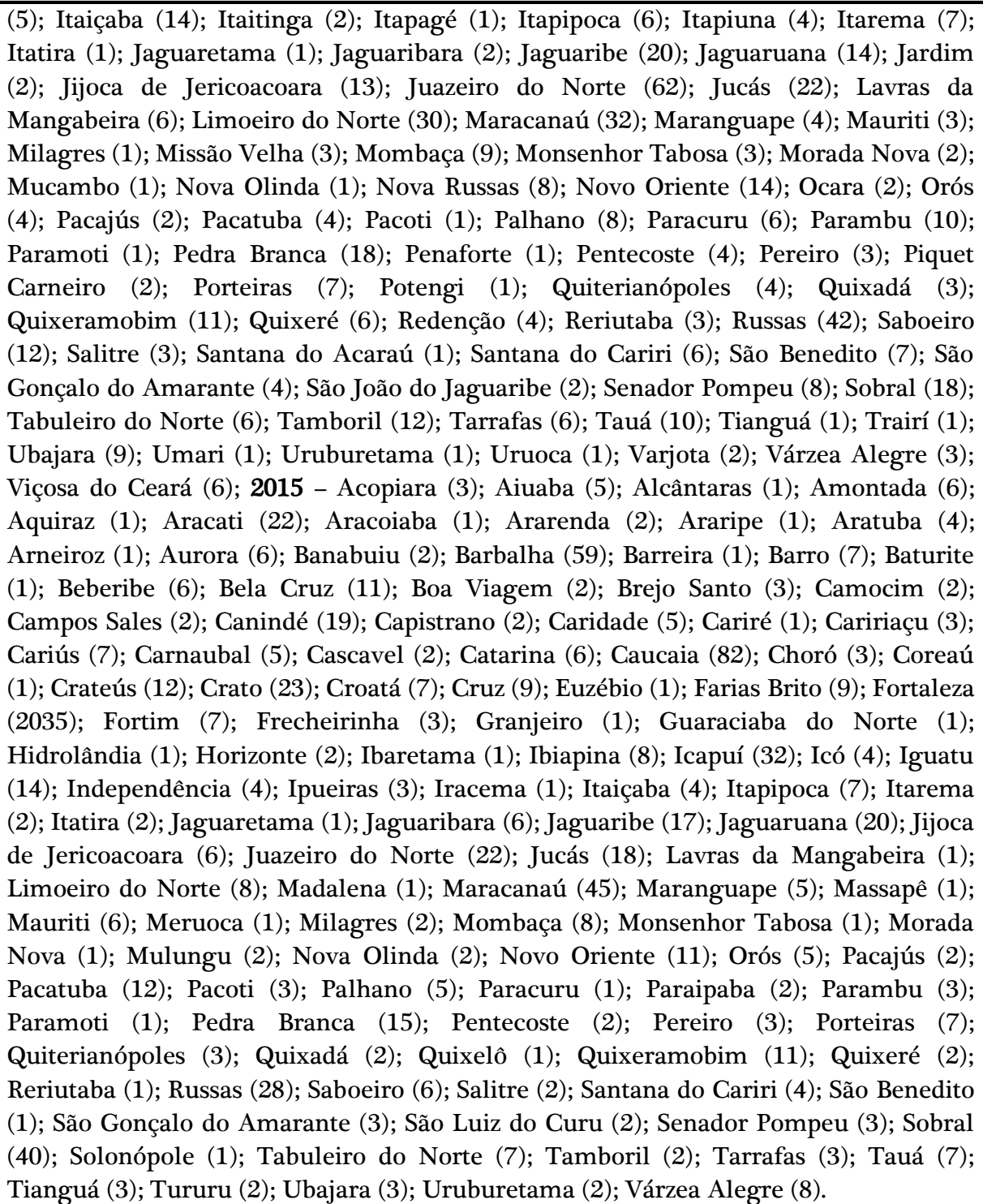 \\
\hline
\end{tabular}

Registros no Estado do Ceará de espécies de interesse em saúde baseados nos espécimes depositados nas coleções de aracnídeos

Cinco espécies de aracnídeos de interesse em saúde são registradas nas coleções examinadas (municípios são destacados em negrito):

\author{
Araneae \\ Ctenidae Keyserling, 1877 \\ Phoneutria Perty, 1833 \\ Phoneutria nigriventer (Keyserling, 1891) \\ (Figura 4A)
}

Material examinado: Brasil. Ceará: Fortaleza (0343'06" S, 38³2'36" O) 1 (IBSP: 1883). 


\section{Loxosceles amazonica Gertsch 1967}

(Figura 4B)

Material examinado: Brasil. Ceará: Aiuaba (06³4'11"S, 4007'28" O): Sítio Pé Queimado: $2{ }^{\circ}$ (IBSP 56059); Gruta Sobradinho: 2 , 2 juvenis (IBSP 154178); 2 , 2 juvenis (IBSP 154181); 2 , 1 juvenil (IBSP 154182); 2 + 1 juvenil (IBSP 154184); 1 ㅇ (IBSP 56061), Estação Ecológica

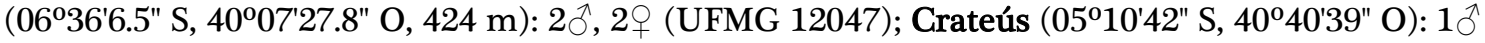
(IBSP 63765); Crato (07014'03" S, 39²4'34" O): 1 ㅇ (IBSP 5979); 4 ( (IBSP 6009); 3 , 2 juvenis (IBSP 11898), Parque Estadual Sítio Fundão (07 13'56.5" S, 39²6'16.8" O, 482 m): 1 ( (UFMG 12064); Parambu, Rodovia Cachoeira do Calista ou Rodovia BR 020 (06²'40" S, 4041'40" O): 1 ㅇ (MNRJ 18415); Pentecostes (03047'34" S, 39016'13" O): Fazenda Experimental da Universidade Federal do Ceará: 1ð⿱ (IBSP 149944); 1 ㅇ, 2 juvenis (IBSP 97966); 1 (IBSP 979667); 3 (IBSP

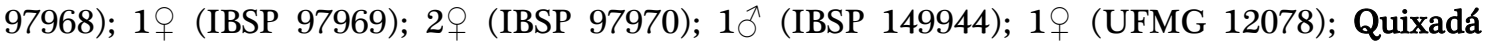
(0458'17" S, 3900'55" O): Fazenda Magé (0456'48.5" S, 39001'22.8" O, 195 m): 1 q (UFMG 12044); Monumento Natural de Monólitos de Quixadá (0455'45.5" S, 3859'18.7" O, 178 m), 1 \% (UFMG

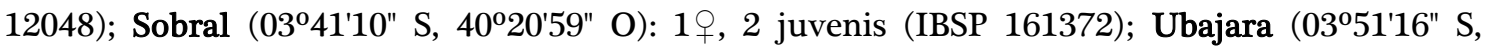
4055'16" O): Gruta do Morcego Branco: 2ㅇ, 4 juvenis (IBSP 152804), Parque Nacional de Ubajara: 2 (IBSP 89326).

Theridiidae Sundevall, 1833

Latrodectus Walckenaer, 1805

Latrodectus aff. curacaviensis Koch, 1841

(Figura 4G)

Material examinado: Brasil. Ceará: Fortaleza (03º̛3'02"S, 38³2'35" W): 1ð̂, 1 (IBSP 14054).

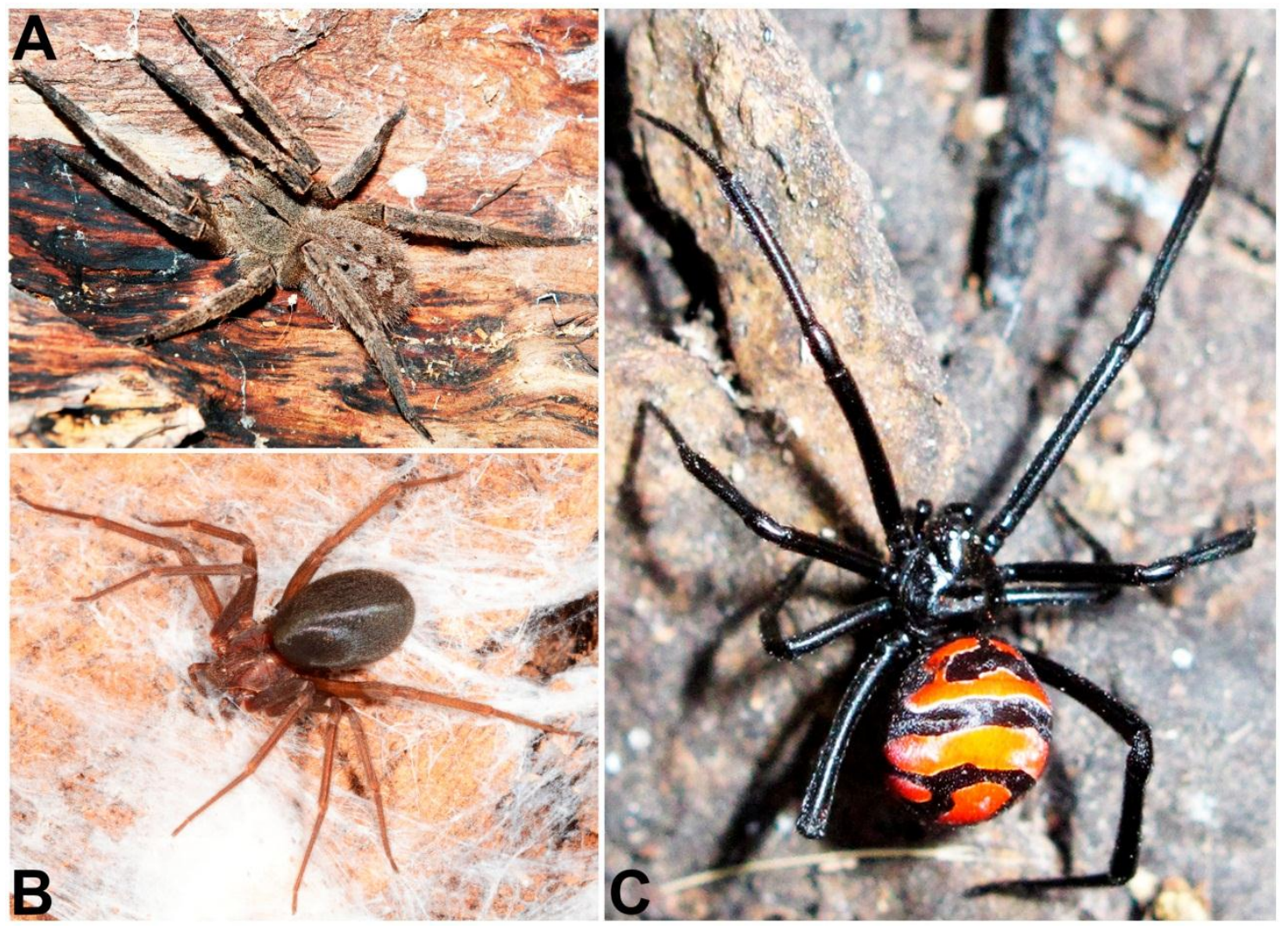

Figura 4. Aranhas de interesse em saúde pública registradas nas coleções examinadas: A. Phoneutria nigriventer, B. Loxosceles amazonica; C. Latrodectus aff. curacaviensis. Fotos: Pedro H. Martins. 


\section{Scorpiones \\ Buthidae C.L. Koch, 1837 \\ Tityus Koch, 1836 \\ Tityus stigmurus (Thorell, 1876) \\ (Figura 5A)}

Material examinado: Brasil. Ceará: Crato (070014'03" S, 39² $24^{\prime} 34^{\prime \prime}$ O) (IBSP 3841); Fortaleza (034'ㅇ' $06^{\prime \prime}$ S, 38 32'36" O) (IBSP 876); Centro de Informação Antiveneno de Fortaleza (IBSP 1550), Secretaria de Saúde de Fortaleza (IBSP 1558), Secretaria de Saúde de Fortaleza (IBSP 1559); CEATOX (IBSP 2733); NUVET (IBSP 6240), (IBSP 6246); Sobral (03²41'10" S, 40²0'59" O), (IBSP $654)$.

Tityus serrulatus (Lutz \& Mello, 1922)

(Figura 5B)

Material examinado: Brazil. Ceará: Fortaleza (03043'06.22" S, 38 32'36.22" O), 1 juvenil (UFMG

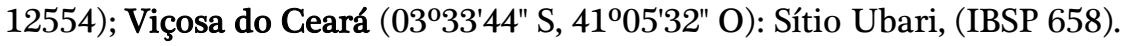

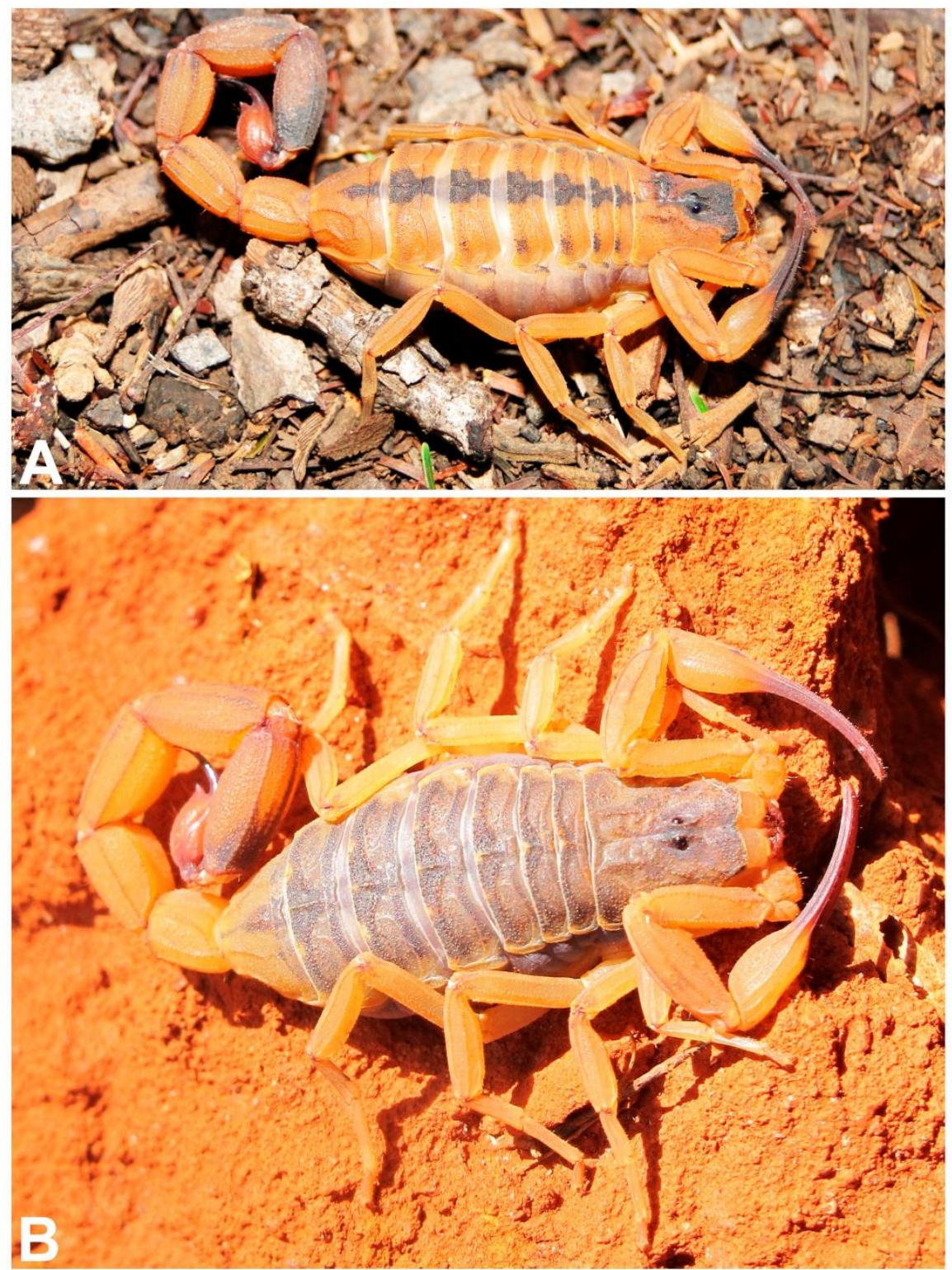

Figura 5. Escorpiões de interesse em saúde pública registrados nas coleções examinadas: A. Tityus stigmurus (Foto: Leonardo S. Carvalho); B. Tityus serrulatus (Foto: Pedro H. Martins). 


\section{Discussão}

Acidentes causados por aranhas e escorpiões são considerados em muitos países um problema de saúde pública (BRASIL 2001) devido à grande probabilidade de acidentes fatais. No Brasil, esses fatores são atrelados a casos subnotificados e com espécies causadoras de acidentes não identificados.

Menos de 5\% dos acidentes causados por aranhas são identificados (Sezerino et al. 1998). Quando um taxonomista ou especialista de outra área identifica a aranha causadora do acidente, a severidade do acidente e eventuais cuidados médicos são facilmente conduzidos e de maneira mais rápida (Isbister \& Gray 2002). Para escorpiões, normalmente a vítima do acidente não coleta o escorpião e nem tampouco o leva para ser identificado. Isso faz com que uma única espécie seja atribuída a diversos acidentes, quando na verdade não ocorre dessa maneira, como é o caso de Tityus stigmurus no Estado de Pernambuco (De Albuquerque et al. 2009).

Talvez exista a mesma tendência para os acidentes atribuídos a Loxosceles no Estado do Ceará, devido à ocorrência de Sicarius Walckenaer, 1847 (Araneae: Sicariidae, mesma família que abriga o gênero Loxosceles), podendo ser responsável por parte dos acidentes atribuídos à aranha-marrom. Apesar de Sicarius não ser considerada uma espécie de interesse em saúde pública e seus acidentes serem incomuns no Brasil, a literatura reporta um acidente causado por Sicarius tropicus (Mello-Leitão, 1936) em um homem de 17 anos, apresentando lesão similar a lesão causada por Loxosceles (Dos Santos \& Cardoso 1992).

As espécies de Sicarius possuem na composição de seu veneno a proteína Sphingomyelinase D (Binford \& Wells 2003). Essa proteína é responsável por causar necrose em suas vítimas (Futrell 1992; Málaque et al. 2002). Sicarius também tem distribuição geográfica atrelada a ambientes xéricos na América Central e do Sul, desertos e florestas tropicais sazonalmente secas (Binford et al. 2008) condições semelhantes as encontradas no Estado do Ceará, onde há registro de duas espécies: Sicarius tropicus em Jaguaribara, Quixadá e Tejuçuoca e Sicarius cariri Magalhães, Brescovit \& Santos, 2013 em Aiuaba, Araripe, Crato e Santana do Cariri (Magalhães et al. 2013). Na macrorregião do Cariri, Sicarius cariri tem sido observada no ambiente externo (quintais) de algumas residências mais afastadas dos municípios do Crato e de Juazeiro do Norte (dados não publicados).

Em acidentes específicos como os causados por Loxosceles, raramente o animal é identificado porque a maioria das pessoas não leva o indivíduo para ser identificado (Vetter 1999; Málaque et al. 2002), principalmente devido a vários dos acidentes ocorrerem quando as pessoas estão dormindo. Essa identificação é feita por meio de dados epidemiológicos e observações clínicas, o que não garante uma identificação acurada da espécie, resultando em falsas informações.

Treze espécies de Loxosceles ocorrem no Brasil, e desse total o Estado do Ceará reporta a ocorrência de Loxosceles amazonica em cavernas e em residências (Azevedo et al. 2014), além de ter registro de acidente para a região sul do Estado (Lucas et al. 1984). Loxosceles amazonica é considerada uma espécie sinantrópica (Carvalho et al. 2014) e ocorre em outros estados da Região Nordeste (Brazil et al. 2009; Silveira 2015).

Dados do SINAN atribuem muitos acidentes a Phoneutria sp., contudo, em todas as coleções consultadas há somente um único espécime de Phoneutria nigriventer, coletado em Fortaleza, oriundo de um caminhão de frutas. Esse espécime não constitui evidência de população estabelecida e, tampouco, pode ser responsável por tantos acidentes como citado na Tabela 2.

Phoneutria nigriventer é uma espécie sinantrópica cuja distribuição original é reportada para as regiões Centro-Oeste e Sudeste do Brasil (Simó \& Brescovit 2001). Os limites ao norte da sua distribuição geográfica se estendem ao Estado da Bahia (Brazil et al. 2009).

É plausível acreditar que existem outras espécies que causem acidentes similares a Phoneutria, como os ctenídeos do gênero Ctenus Walckenaer, 1805. Ctenus medius Keyserling, 1891 é facilmente confundida com as Phoneutria spp., principalmente com os machos adultos devido ao seu padrão de coloração e tamanho (Almeida et al. 2000), e propriedades toxicológicas 
similares, principalmente a dor intensa ou hiperalgesia (Okamoto et al. 2009), cujo sintoma é o caráter que define o acidente causado por Phoneutria sp. (Camplesi et al. 2014). Essa suposição se justifica nos registros de Ctenus rectipes F.O. Pickard-Cambridge, 1897 em municípios da macrorregião Sobral (Brescovit \& Simó 2007).

Tityus stigmurus é uma espécie endêmica da região Nordeste (BRASIL 2009; De Souza et al. 2009), sendo a principal responsável pelos acidentes na região (Porto \& Brazil 2010).

Tityus serrulatus é uma espécie exótica e o principal escorpião responsável por causar acidentes no Brasil (Porto \& Brazil 2010). A distribuição geográfica de T. serrulatus contempla as regiões Nordeste e Centro-Oeste, dentre elas, o Estado do Ceará (BRASIL 2009) e, mais recentemente, a região Sul (Da Rosa et al. 2015). No interior do Ceará, T. serrulatus é encontrado em áreas urbanas e áreas de Caatinga (Porto et al. 2014).

Devido à complexidade em se identificar espécies do complexo Tityus stigmurus, $T$. serrulatus apresenta uma distribuição geográfica que necessita ser analisada com parcimônia em virtude de todas as sinonímias que foram feitas com a espécie (De Souza et al. 2009 para mais detalhes). Desse modo, algumas localidades não apresentam ocorrência de $T$. serrulatus. A exemplo disto, Lourenço \& Cloudsley-Thompson (1999) identificaram equivocadamente exemplares de $T$. melici como sendo $T$. serrulatus. Populações dessa espécie são reportadas para os Estados da Bahia e Minas Gerais (De Souza et al. 2009).

Uma das razões para a escassez de informações sobre aranhas e escorpiões de interesse em saúde pública no Estado do Ceará é a ausência de centros de pesquisa e, consequentemente, a subnotificação dos dados. Todas as informações obtidas e citadas nesse artigo quando combinadas podem produzir estratégias para minimizar o número de acidentes e/ou melhorar as respostas dadas aos acidentes com aracnídeos.

A tabulação dos dados obtidos a partir das coleções científicas reconhecidas e dos acidentes nas macrorregiões citadas demonstra a discrepância entre as informações obtidas pelas autoridades de saúde. O significado dessa discrepância vai de encontro a ausência de um especialista no pronto atendimento e a inconstância de dados taxonômicos e epidemiológicos da ficha do SINAM, onde a identificação do agente causador do acidente não carece da presença de um taxonomista no ambiente hospitalar. Esta ausência corrobora em dados totalmente subnotificados. Apenas com a verificação em coleções zoológicas, nota-se o erro quanto a identificação taxonômica e ocorrência de animais sinantópicos em distintos biomas.

Outro fato que agrava as subnotificações em áreas populosas é a ausência da obrigatoriedade de encaminhar o agente causador do acidente a um especialista para que o mesmo possa ser identificado e inserido na coleção de referência.

Políticas de saúde pública devem ser revistas com o objetivo de melhorar o atendimento clínico, dados com embasamento técnico-científico e fomentar dados estatísticos de distribuição geográfica de animais sinantrópicos, particularmente, aranhas e escorpiões.

Desse modo, esperamos que novos trabalhos sobre a história natural de aranhas e escorpiões no Estado do Ceará sejam desenvolvidos, bem como estudos de distribuição geográfica e epidemiológicos.

\section{Agradecimentos}

Aos avaliadores anônimos pela revisão crítica do manuscrito.

\section{Referências}

Acidentes por animais peçonhentos (2017) Portal da Saúde. Disponível em: http://portalsaude.saude.gov.br/index.php/o-ministerio/principal/secretarias/svs/acidentes-poranimais-peconhentos (Acessado em 19/10/2017).

Almeida C.E., Ramos E.F., Gouvêa E., Carmo-Silva M. \& Costa J. (2000) Natural history of Ctenus medius Keyserling, 1891 (Aranae, Ctenidae): observations on habitats and the development of chromatic patterns. Revista Brasileira de Biologia, 60: 503-509. 
Alves R.S., Martins R.D., Sousa D.F., Alves C.D., Barbosa P.S.F., Queiroz M.G.R.Q., Martins A.M.C. \& Monteiro H.S.A. (2007) Aspectos epidemiológicos dos acidentes escorpiônicos no estado do Ceará no período de 2003 a 2004. Revista Eletrônica Pesquisa Médica, 1(3): 14-20.

Azevedo R., Texeira P.M.S., Siqueira R.C.L. \& Brescovit A.D. (2014) New record and distribution of Loxosceles amazonica Gertsch, 1967 (Araneae: Sicariidae) in the State of Ceará, Brazil. Check List, 10(1): 207-208.

Binford G.J. \& Wells M.A. (2003) The phylogenetic distribution of sphingomyelinase D activity in venoms of haplogyne spiders. Comparative Biochemistry and Physiology (Part B), 135: 125-133.

Binford G.J., Callahan M.S., Bodner M.R., Rynerson M.R., Núñez P.B., Ellison C.E. \& Duncan R.P. (2008) Phylogenetic relationships of Loxosceles and Sicarius spiders are consistent with Western Gondwanan vicariance. Molecular Phylogenetics and Evolution, 49: 538-553.

BRASIL (2001) Manual de Diagnóstico e Tratamento de Acidentes por Animais Peçonhentos. $2^{\circ}$ edição. Brasília: Fundação Nacional de Saúde. 112 p.

BRASIL (2009) Manual de Controle de Escorpiões. Brasília: Ministério da Saúde. 74 p.

Brazil T.K. \& Porto T.J. (2010) Os escorpiões. Salvador: EdUFBA. 90 p.

Brazil T.K., Pinto-Leite C.M., Almeida-Silva L.M., Lira-Da-Silva R.M. \& Brescovit A.D. (2009) Aranhas de importância médica do Estado da Bahia. Gazeta Médica da Bahia, 79(Supl.1): 3237.

Brecovit A.D. \& Simó M. (2007) On the Brazilian Atlantic Forest species of the spider genus Ctenus Walckenaer, with the description of a neotype for C. dubius Walckenaer (Araneae, Ctenidae, Cteninae). Bulletin of British Arachnology Society, 14: 1-17.

Camplesi A.C., Albernaz S.S., Burger K.P. \& Araújo C.F.M. (2014) Accidents Caused by Spider Bites. Open Journal of Animal Science, 4: 113-117.

Carvalho L.S., Brescovit A.D., Santos A.J., Oliveira U. \& Guadanucci J.P.L. (2014) Aranhas da Caatinga (p. 15-33). In: Bravo F. \& Calor A. (Eds) Artrópodes do semi-árido: biodiversidade e conservação. Feira de Santana: Printmídia. 298 p.

Ceará (2010) Coordenadorias Regionais de Saúde. Disponível em: http://www.saude.ce.gov.br/index.php/regionalizacao (Acessado em: 05/05/2017).

Da Rosa C.M., Abegg A.D., Borges L.M., Bitencourt G.S.S. \& Di Mare R.A. (2015) New record and occurrence map of Tityus serrulatus Lutz \& Mello, 1922 (Scorpiones, Buthidae) in the state of Rio Grande do Sul, Southern Brazil. Check List, 11(1): 1-3.

De Albuquerque C.M.R., Porto T.J., Amorim M.L.P. \& Santana Neto P.L. (2009) Escorpionismo por Tityus pusillus Pocock, 1893 (Scorpiones; Buthidae) no Estado de Pernambuco. Revista da Sociedade Brasileira de Medicina Tropical, 42(2): 206-208.

De Souza, C.A.R., Candido D.M., Lucas S.M. \& Brescovit A.D. (2009) On the Tityus stigmurus complex (Scorpiones, Buthidae). Zootaxa, 1987: 1-38.

Dos Santos M.C. \& Cardoso J.L.C (1992) Lesão dermonecrótica por Sicarius tropicus, simulando loxoscelismo cutâneo. Revista da Sociedade Brasileira de Medicina Tropical, 25: 115-123.

Furtado S.S., Belmino J.F.B., Diniz A.G.Q. \& Leite R.S. (2016) Epidemiology of scorpion envenomation in the state of Ceará, Northeastern Brazil. Revista do Instituto de Medicina Tropical São Paulo, 58(15): 1-5.

Futrell J.M. (1992) Loxoscelism. The American Journal of Medical Sciences, 304: 261-267.

Gertsch W.J. (1967) The spider genus Loxosceles in South America (Araneae, Scytodidae). Bulletin of American Museum of Natural History, 136: 117-174.

IBGE (2010) Instituto Brasileiro de Geografia e Estatística. Disponível em: http://www.ibge.gov.br/home/estatistica/populacao/censo2010 (Acessado em: 07/04/2017).

Isbister G.K. \& Gray M.R. (2002) A prospective study of 750 definite spider bites, with expert spider identification. Quarterly Journal of Medicine, 95: 723-731.

Levi H.W. (1959) The spider genus Latrodectus (Araneae, Theridiidae). Transations of American Microscopic Society, 78: 07-43. 
Lourenço W.R. \& Cloudsley-Thompson J.L. (1999) Discovery of a sexual population of Tityus serrulatus, one of the morphs within the complex Tityus stigmurus (Scorpiones, Buthidae). The Journal of Arachnology, 27: 154-158.

Lourenço W.R. \& Eickstead V.R.D. (2003) Escorpiões de Importância Médica (p. 182-197). In: Cardoso J.L.C., França F.O.S., Wen F.H., Málaque C.M.S. \& Haddad V. (Eds). Animais Peçonhentos no Brasil: biologia, clínica e terapêutica dos acidentes. São Paulo: Sarvier. 468 p.

Lucas S.M., Cardoso J.L.C. \& Moraes A.C. (1984) Loxoscelismo: relato de um acidente humano atribuído a Loxosceles amazonica Gertsch, 1967 (Araneae, Scytodidae, Loxoscelinae). Memorias do Instituto Butantan, 47/48: 127-131.

Magalhães I.L.F., Brescovit A.D. \& Santos A.J. (2013) The six-eyed sand spiders of the genus Sicarius (Araneae: Haplogynae: Sicariidae) from the Brazilian Caatinga. Zootaxa, 3599 (2): 101-135.

Málaque C.M.S., Castro-Valencia J.E., Cardoso J.L.C., França F.O.S., Barbaro K.C. \& Fan H.W. (2002) Clinical and epidemiological features of definitive and presumed loxoscelism in São Paulo, Brazil. Revista do Instituto de Medicina Tropical São Paulo, 44: 139-143.

Martins R. \& Bertani R. (2007) The non-Amazonian species of the Brazilian wandering spiders of the genus Phoneutria Perty, 1833 (Araneae: Ctenidae), with the description of a new species. Zootaxa, 1526: 1-36.

Nimer E. (1972) Climatologia da região Nordeste do Brasil: introdução à climatologia dinâmica. Revista Brasileira de Geografia, 34: 03-51.

Okamoto C.K., Gonçalves-De-Andrade R.M., Queiroz G.P., Gutierez V.P., De Almeida D.M., Cury Y., Bertani R., Portaro F.C. \& Tambourgi D.V. (2009) Ctenus medius and Phoneutria nigriventer spiders venoms share noxious proinflammatory activities. Journal of Medical Entomology, 46: 58-66.

Porto T.J. \& Brazil T.K. (2010) Os escorpiões de importância médica e seus venenos (p. 65-75). In: Brazil T.K. \& Porto T.J. (Eds). Os escorpiões. Salvador: Edufba. 90 p.

Porto T.J., Carvalho L.S., Souza C.A.R., Oliveira U. \& Brescovit A.D. (2014) Escorpiões da Caatinga: conhecimento atual e desafios (p. 33-47). In: Bravo F. \& Calor A. (Eds). Artrópodes do Semi-Árido: biodiversidade e conservação. Feira de Santana: Printmídia. 298 p.

Rein J.O. (2012) The Scorpion Files. Trondheim: Norwegian University of Science and Technology. Disponível em: http://www.ntnu.no/ub/scorpion-files (Acessado em: 05/05/2017).

Reis A.C. (1976) Clima da caatinga. Anais da Academia Brasileira de Ciências, 48: 325-335.

Sezerino U.M., Zannin M., Coelho L.K., Gonçalves Júnior J., Grando M., Mattosinho S.G., Cardoso J.L., Von Eickstedt V.R., França F.O., Barbaro K.C. \& Fan H.W. (1998) A clinical and epidemiological study of Loxosceles spider envenoming in Santa Catarina, Brazil. Transations of Royal Society of Tropical Medicine and Hygiene, 92: 546-548.

Silveira A.L. (2015) Novos registros geográficos da aranha-marrom Loxosceles amazonica Gertsch, 1967 (Araneae, Sicariidae) no Nordeste do Brasil e sua importância médica. Revista Médica Minas Gerais, 25 (1): 37-45.

Simó M. \& Brescovit A.D. (2001) Revision and cladistic analysis of the Neotropical spider genus Phoneutria Perty, 1833 (Araneae, Ctenidae), with notes on related Cteninae. Bulletin of British Arachnology Society, 12(2): 67-82.

SINAN (2017) Sistema de Informação de Agravos de Notificação. Disponível em: http://portalSINAN.saude.gov.br/dados-epidemiologicos-SINAN (Acessado em: 08/05/2017).

Vetter R.S. (1999) Identifying and misidentifying the brown recluse spider. Dermatology Online Journal, 5(2): 1-7.

World Spider Catalog (2017) Natural History Museum Bern. Disponível em: http://wsc.nmbe.ch. http://wsc.nmbe.ch (Acessado em: 04/10/2017). 